\title{
Tachyonic Teleparallel Dark Energy in Phase Space
}

\author{
Behnaz Fazlpour ${ }^{1}$ and Ali Banijamali ${ }^{2}$ \\ ${ }^{1}$ Department of Science, Babol Branch, Islamic Azad University, Babol, Iran \\ ${ }^{2}$ Department of Basic Sciences, Babol University of Technology, Babol 47148-71167, Iran
}

Correspondence should be addressed to Behnaz Fazlpour; b.fazlpour@umz.ac.ir

Received 24 August 2013; Accepted 23 September 2013

Academic Editor: Kadayam S. Viswanathan

Copyright (C) 2013 B. Fazlpour and A. Banijamali. This is an open access article distributed under the Creative Commons Attribution License, which permits unrestricted use, distribution, and reproduction in any medium, provided the original work is properly cited.

\begin{abstract}
Recently, nonminimal coupling between a noncanonical scalar field and gravity in the framework of teleparallelism has been proposed. Noncanonical scalar field is tachyon field, and the model is known as tachyonic teleparallel dark energy. Here, we perform a dynamical analysis of the model, find its critical points, and study their stability. We find that all the critical points are dark energy dominated solutions corresponding to an accelerating universe. It is also shown that there exist two critical lines which are stable attractors of the model.
\end{abstract}

\section{Introduction}

Recent accelerated expansion of our universe is one of the most significant cosmological discoveries over the last decade [1-6]. There are two possibilities to explain such a behavior. One possibility in explaining the observations is by assuming that at large scales the Einstein theory of general relativity breaks down, and a more general action describes the gravitational field. The well-known $f(R)$ modified gravity belongs to this sort of possibilities in which one replaces the Ricci scalar $R$ in the Einstein-Hilbert action by an arbitrary function of $R$ (for reviews see [7-10]).

The other approach is modifying the content of the universe by introducing dark energy component with negative pressure whose nature and cosmological origin still remain enigmatic at present. The simplest candidate of dark energy is the cosmological constant, but it suffers from two kinds of problems: fine tuning and coincidence problem [11].

A dynamical scalar field with tachyon, quintessence, or phantom behavior or a combination of the last two fields in a unified scenario called quintom [12-18] is another proposed for dark energy (for reviews see [19-22]).

In a different approach, avoiding the curvature defined via the Levi-Civita connection, one could explore an alternative way and use the Weitzenböck connection that has no curvature but instead torsion. This has the property that the torsion is formed completely from products of first derivatives of the tetrad, with no second derivatives appearing in the torsion tensor. This approach was considered originally by Einstein in 1928 [23-29], as teleparallelism. It is closely related to standard general relativity, differing only in "boundary terms" involving total derivatives in the action [30]. Inspired from $f(R)$ gravity, the so-called $f(T)$ gravity (generalization of the teleparallel gravity) has recently drawn much attention [3136].

Using the teleparallel equivalent of general relativity as the gravitational sector, recently Geng et al. have proposed teleparallel dark energy by adding a canonical scalar field, allowing for a nonminimal coupling with gravity [37, 38]. They have used the quintessence scalar field as being responsible for dark energy. Although teleparallel dark energy scenario in the minimal case is completely equivalent with standard quintessence, it shows interesting cosmological behavior in the nonminimal case. Such a model exhibits quintessence-like or phantom-like behavior and also realizes crossing of the phantom divide line [37,38].

In [39], we have studied the tachyonic teleparallel dark energy where instead of quintessence we used tachyon scalar field responsible for dark energy. Such a model can also realize the phantom divide crossing. In this paper, we present a phase space analysis of the above-mentioned scenario.

One important test for dark energy models is the investigation of their phase space analysis. In such analysis, one 
examines whether dark energy models have attractor solutions corresponding to accelerating universe and ration of $\Omega_{\mathrm{DE}} / \Omega_{\mathrm{DM}}$ of order 1 . If these conditions are fulfilled, then the universe will result in that solution at late time, independently of the initial conditions, and the basic observational requirements will be satisfied [40]. Phase space analysis of various cosmological models have been studied by different authors (e.g., see [41-48]).

Phase space analysis of the original teleparallel dark energy has been done in [47]. The authors have found that this scenario possesses a late time attractor in which dark energy behaves like a cosmological constant independently of the specific values of the model parameter, and so this feature provides a natural way for the stabilization of the dark energy equation of state parameter to the cosmological constant value, without the need for parameter tuning. Here, we find the critical solutions of the tachyonic teleparallel dark energy model.

An outline of the present work is as follows. In Section 2, we briefly review our model by obtaining the energy density as well as the pressure and equation of state parameter of dark energy. In Section 3, we study the phase space analysis of the model using the system of autonomous differential equations, find its fixed points, and study their stability. Section 4 is devoted to conclusions.

\section{The Model}

We start with a brief review of teleparallel gravity. In such theory, orthonormal tetrad components $e_{i}\left(x^{\mu}\right)$ are used, where an index $i$ runs over $0,1,2,3$ for the tangent space at each point $x^{\mu}$ of the manifold [23-29, 49-54]. They are satisfied in the following relation:

$$
g_{\mu \nu}=\eta_{i j} e_{\mu}^{i} e_{\nu}^{j}
$$

where $\mu$ and $\nu$ are coordinate indices on the manifold, take the values $0,1,2,3$. The Lagrangian of teleparallel gravity is given by the torsion scalar $T$ which is defined as

$$
T \equiv S_{\rho}^{\mu \nu} T_{\mu \nu}^{\rho}
$$

where the torsion $T_{\mu \nu}^{\rho}$ and contorsion $K_{\rho}^{\mu \nu}$ and $S_{\rho}^{\mu \nu}$ are defined by

$$
\begin{gathered}
T_{\mu \nu}^{\rho} \equiv e_{i}^{\rho}\left(\partial_{\mu} e_{\nu}^{i}-\partial_{\nu} e_{\mu}^{i}\right), \\
K_{\rho}^{\mu \nu} \equiv-\frac{1}{2}\left(T_{\rho}^{\mu \nu}-T_{\rho}^{\nu \mu}-T_{\rho}^{\mu \nu}\right), \\
S_{\rho}^{\mu \nu} \equiv \frac{1}{2}\left(K_{\rho}^{\mu \nu}+\delta_{\rho}^{\mu} T_{\theta}^{\theta \nu}-\delta_{\rho}^{\nu} T_{\theta}^{\theta \mu}\right) .
\end{gathered}
$$

We recall that the Lagrangian density of general relativity is described by the Ricci scalar $R$. So, our starting action in teleparallelism is as follows:

$$
S=\int d^{4} x e\left[\frac{T}{2 \kappa^{2}}+\mathscr{L}_{m}\right]
$$

where $e=\operatorname{det}\left(e_{\mu}^{i}\right)=\sqrt{-g}$ and $\kappa^{2}=8 \pi G=1 / M_{\mathrm{Pl}}^{2}$ while $G$ is a bare gravitational constant and $M_{\mathrm{Pl}}$ is the reduced
Planck mass. The above scenario cannot describe an accelerated universe. So, we should generalize the action (4). The generalization can be categorized in two ways: using a general function $f(T)$ instead of $T$ in action (4) which is inspired by the well-known $f(R)$ gravity [24-29, 37, 55-57] or adding a scalar field responsible for dark energy in (4). Note that in the second approach one can also allow a nonminimal coupling between the scalar field and gravity.

In this paper, we consider the following action as an example of the second approach:

$$
\begin{aligned}
S=\int d^{4} x e[ & \frac{T}{2 \kappa^{2}}+f(\phi) T \\
& \left.-V(\phi) \sqrt{1+g^{\mu \nu} \partial_{\mu} \phi \partial^{\nu} \phi}+\mathscr{L}_{m}\right],
\end{aligned}
$$

where $f(\phi)$ is an arbitrary function of scalar field and it is responsible for nonminimal coupling between dark energy and gravity. The last term in the above action is the BornInfeld-type action for tachyon field, where $V(\phi)$ is the tachyonic potential which is bounded and reaches its minimum asymptotically. We should emphasize that in torsion formulation of general relativity the only scalar is the torsion, and hence the nonminimal coupling will be between this and tachyon field in analogy with the standard nonminimal tachyon cosmology in general relativity where the scalar field couples to the Ricci scalar.

In a spatially-flat Friedmann-Robertson-Walker (FRW) space-time,

$$
d s^{2}=-d t^{2}+a^{2}(t)\left(d r^{2}+r^{2} d \Omega^{2}\right),
$$

where $t$ is the cosmic time, $a(t)$ is the scale factor and a vierbein choice of the form $e_{\mu}^{i}=\operatorname{diag}(1, a, a, a)$; the corresponding Friedmann equations are given by

$$
\begin{gathered}
H^{2}=\frac{\kappa^{2}}{3}\left(\rho_{\phi}+\rho_{m}\right), \\
\dot{H}=-\frac{\kappa^{2}}{2}\left(\rho_{\phi}+P_{\phi}+\rho_{m}+P_{m}\right),
\end{gathered}
$$

where $H=\dot{a} / a$ is the Hubble parameter and a dot stands for the derivative with respect to $t$. In these equations, $\rho_{m}$ and $P_{m}$ are the matter energy density and pressure, respectively. They follow the standard evolution equation $\dot{\rho}_{m}+3 H(1+$ $\left.\omega_{m}\right) \rho_{m}=0$, with $\omega_{m}=P_{m} / \rho_{m}$ being the matter equation of state parameter. The effective energy density and pressure of tachyonic teleparallel dark energy read

$$
\begin{aligned}
\rho_{\phi}= & \frac{V(\phi)}{\sqrt{1-\dot{\phi}^{2}}}-6 H^{2} f(\phi), \\
P_{\phi}= & -V(\phi) \sqrt{1-\dot{\phi}^{2}}+2\left(3 H^{2}+2 \dot{H}\right) f(\phi) \\
& +2 H f^{\prime}(\phi) \dot{\phi}\left(1+\frac{1}{\sqrt{1-\dot{\phi}^{2}}}\right),
\end{aligned}
$$

where a prime denotes derivative with respect to $\phi$. 
Furthermore, the equation of motion of the scalar field in FRW background (6) can be obtained by variation of the action with respect to $\phi$ :

$$
\frac{\ddot{\phi}}{1-\dot{\phi}^{2}}+3 H \dot{\phi}+\frac{V^{\prime}(\phi)}{V(\phi)}+\frac{6 H^{2} f^{\prime}(\phi)}{V(\phi)}=0 .
$$

Note that in (9) and (10) we have used the useful relation:

$$
T=-6 H^{2},
$$

which simply arises from the calculation of (2) for the FRW metric (6). One can see that the scalar field evolution (10) expresses the standard relation for the energy conservation:

$$
\dot{\rho}_{\phi}+3 H\left(1+\omega_{\phi}\right) \rho_{\phi}=0
$$

where $\omega_{\phi}=P_{\phi} / \rho_{\phi}$ is the equation of state parameter of dark energy which is attributed to the scalar field $\phi$.

Tachyonic teleparallel dark energy has been studied in [39]. It is shown that such a scenario can realize phantom divide crossing during its evolution and so exhibits very interesting cosmological behavior. Therefore, it seems necessary to perform a phase-space analysis of such theory. Note that in the original teleparallel dark energy of [37], although the scalar field is canonical, one can obtain a dark energy sector being quintessence-like, phantom-like, or experiencing the phantom-divide crossing during evolution. The fact that the phantom regime is possible without the need of phantom fields, which have ambiguous quantum behavior [58], is a significant advantage. In phase space analysis, we investigate late-time solutions that are independent of the initial conditions and the specific universe evolution. From here, we consider a nonminimal coupling function of the form $f(\phi)=(1 / 2) \xi \phi^{2}$.

\section{Phase Space Analysis}

In order to study the phase space and stability of the model (1), we should translate the evolution equations in the language of the autonomous dynamical system $X^{\prime}=f(X)[40,59-$ 62 ], where $X$ is the column vector constituted by suitable auxiliary variables, $f(X)$ is the corresponding column vector of the autonomous equations, and prime denotes derivative with respect to the logarithm of the scale factor $N=\ln a$. The critical points $X_{c}$ are extracted from $X^{\prime}=0$, and in order to determine the stability properties of these critical points we expand around $X_{c}$, setting $X=X_{c}+U$ with $U$ the perturbations of the variables considered as column vector. Thus, up to the first order we acquire $U^{\prime}=Q \cdot U$, where the matrix $Q$ contains the coefficients of the perturbation equations. For each critical point, the eigenvalues of $Q$ determine its type and stability.
Now, let us transform the cosmological equations into an autonomous dynamical system. To this end, we introduce the following auxiliary variables:

$$
x=\frac{\kappa \sqrt{V(\phi)}}{\sqrt{3} H}, \quad y=\dot{\phi}, \quad z=\kappa \phi \sqrt{|\xi|}, \quad u=\frac{\kappa}{H}
$$

By rewriting the Friedmann equation (7) in terms of these variables, we reach a constraint that allows expressing $\rho_{m}$ as a function of the auxiliary variables as follows:

$$
\frac{x^{2}}{\sqrt{1-y^{2}}}-z^{2} \operatorname{sgn}(\xi)+\frac{\kappa^{2} \rho_{m}}{3 H^{2}}=1 \text {. }
$$

Furthermore, the auxiliary variables allow us to straight forwardly obtain the density parameter of dark energy and dark matter as

$$
\begin{gathered}
\Omega_{m} \equiv \frac{\kappa^{2} \rho_{m}}{3 H^{2}}=1-\frac{x^{2}}{\sqrt{1-y^{2}}}+z^{2} \operatorname{sgn}(\xi), \\
\Omega_{\mathrm{DE}} \equiv \frac{\kappa^{2} \rho_{m}}{3 H^{2}}=\frac{x^{2}}{\sqrt{1-y^{2}}}-z^{2} \operatorname{sgn}(\xi) .
\end{gathered}
$$

While the equation of state of the field reads

$$
\begin{aligned}
& \omega_{\mathrm{DE}} \equiv \omega_{\phi}=\frac{P_{\phi}}{\rho_{\phi}} \\
& =\left(-x^{2} \sqrt{1-y^{2}}-z^{2} \omega_{m} \operatorname{sgn}(\xi)\right. \\
& \times\left[1-\frac{x^{2}}{\sqrt{1-y^{2}}}+z^{2} \operatorname{sgn}(\xi)\right] \\
& \left.+\frac{2}{3} u y z \sqrt{|\xi|} \operatorname{sgn}(\xi)\left(1+\frac{1}{\sqrt{1-y^{2}}}\right)\right) \\
& \times\left(\left[1+z^{2} \operatorname{sgn}(\xi)\right]\right. \\
& \left.\times\left[\frac{x^{2}}{\sqrt{1-y^{2}}}-z^{2} \operatorname{sgn}(\xi)\right]\right)^{-1} .
\end{aligned}
$$

It is shown in [39] that $\omega_{\mathrm{DE}}$ can realize phantom divide crossing during the cosmological evolution. Without loss of generality, in the following we restrict ourselves in the dust matter case; that is, we assume $\omega_{m}=0$. 
Another two additional quantities with great physical significance, namely, the total equation of state parameter and the deceleration parameter are given by

$$
\begin{aligned}
& \omega_{\text {tot }} \equiv \frac{P_{\phi}}{\rho_{\phi}+\rho_{m}} \\
& =\left(-x^{2} \sqrt{1-y^{2}}\right. \\
& \left.+\frac{2}{3} u y z \sqrt{|\xi|} \operatorname{sgn}(\xi)\left(1+\frac{1}{\sqrt{1-y^{2}}}\right)\right) \\
& \times\left(\left[1+z^{2} \operatorname{sgn}(\xi)\right]\right)^{-1}, \\
& q \equiv-1-\frac{\dot{H}}{H^{2}}=\frac{1}{2}+\frac{3}{2} \omega_{\text {tot }} \\
& =\left(1-3 x^{2} \sqrt{1-y^{2}}\right. \\
& \left.-z\left[z+2 u y \sqrt{|\xi|}\left(1+\frac{1}{\sqrt{1-y^{2}}}\right)\right] \operatorname{sgn}(\xi)\right) \\
& \times\left(2\left[1+z^{2} \operatorname{sgn}(\xi)\right]\right)^{-1} \text {. }
\end{aligned}
$$

We mention that relations (17) are always valid, that is, independently of the specific state of the system (they are valid in the whole phase-space and not only at the critical points).

Finally, we should be concerned with the scalar potential $V(\phi)$ in order to handel the potential derivative that is present in (10). We assume a potential of the form

$$
V(\phi)=\alpha \phi^{-2}
$$

Note that such an inverse square potential was used repeatedly as an example in the literature related to tachyon cosmology. The phase plane analysis of tachyon dynamics within FRW cosmology with inverse square potential has been extensively studied in $[63,64]$. Tachyon dynamics for inverse square potential in loop quantum cosmology has been investigated in [65]. It is proven that there exist up to five fixed points with only two of them being possibly stable. Based on a redefinition of tachyon field, [41] has been explored tachyon dynamics for a wide class of self-interaction potentials beyond the inverse square one. It is revealed that for power law potentials the late time attractor is always the de Sitter solution while for sinh-like potentials the late time attractor can be either inflationary tachyon dominated solution or the matter scaling phase.
Now, by inserting the auxiliary variables (13) into the equations of motion (7), (8), and (10) and considering the potential (18), we obtain the following result in our setup:

$$
\begin{aligned}
x^{\prime}= & -\sqrt{|\xi|} x y u z^{-1}+\frac{3}{2} x-\frac{3 x^{3} \sqrt{1-y^{2}}}{2+2 z^{2} \operatorname{sgn}(\xi)} \\
& +\frac{x y z u \sqrt{|\xi|} \operatorname{sgn}(\xi)}{1+z^{2} \operatorname{sgn}(\xi)}\left(1+\frac{1}{\sqrt{1-y^{2}}}\right), \\
y^{\prime}= & -\left(1-y^{2}\right)\left[3 y-2 \sqrt{|\xi|} u z^{-1}+2 \sqrt{|\xi|} u z x^{-2}\right], \\
z^{\prime}= & \sqrt{|\xi| y u,} \\
u^{\prime}= & \frac{3}{2} u-\frac{3 x^{2} u \sqrt{1-y^{2}}}{2+2 z^{2} \operatorname{sgn}(\xi)} \\
& +\frac{y z u^{2} \sqrt{|\xi|} \operatorname{sgn}(\xi)}{1+z^{2} \operatorname{sgn}(\xi)}\left(1+\frac{1}{\sqrt{1-y^{2}}}\right) .
\end{aligned}
$$

Now, let us proceed to the phase space analysis. The critical or fixed points $\left(x_{c}, y_{c}, z_{c}, u_{c}\right)$ of the autonomous system are obtained by setting the left-hand sides of the equations to zero, namely, by imposing the conditions $x^{\prime}=$ $y^{\prime}=z^{\prime}=u^{\prime}=0$.

After some algebraic calculus, we find four critical points $(A, B, C, D)$ and two critical lines $\mathscr{L}_{1}, \mathscr{L}_{2}$ in our model which we have presented in Table 1 . In the same table, we provide their existence conditions. Also, we calculate the values of $\Omega_{\mathrm{DE}}, \omega_{\mathrm{DE}}, \omega_{\mathrm{tot}}$, and $q$ which can be used to discuss whether there exists acceleration phase or not. The $4 \times 4$ matrix $Q$ of the linearized perturbation equations of the autonomous system is shown in the appendix. Therefore, for each critical point of Table 1, we examine the sign of the real part of the eigenvalues of $Q$, namely, $v_{1}, v_{2}, v_{3}$, and $v_{4}$ which determine the type and stability of this specific critical point. A fixed point is unstable if $v_{1}>0, v_{2}>0, v_{3}>0$, and $v_{4}>0$. It is saddle if real part of eigenvalues has different signs and it is stable for negative real part. In Table 2, we have shown the eigenvalues linearization matrix of the fixed points and the critical lines. The behavior of the eigenvalues is presented in Table 3.

3.1. Critical Point A. Point $A$ is a dark energy dominated solution $\left(\Omega_{\mathrm{DE}}=1\right)$ with equation of state-like cosmological constant $\omega_{\mathrm{DE}}=-1$, that exists for negative values of $\xi$. Accelerated expansion occurs for this point because $\omega_{\text {tot }}<$ $-1 / 3$. Existence of the point $A$ is independent of the value of the variable $u$. Depending on the values of $\lambda_{1}, \lambda_{2}$, and $\widehat{\xi}$, this solution can be stable, unstable, or saddle point. For instance, point $A$ is a saddle point (meaning that the universe during its evolution can reach this state but does not remain there and evolves to another state) if nonvanishing eigenvalues of 
TABLE 1: Location and condition for existence of the critical points and the corresponding values of the dark energy density parameter $\Omega_{\mathrm{DE}}$, of dark energy equation of state parameter $\omega_{\mathrm{DE}}$, of the total equation of state parameter $\omega_{\text {tot }}$, and of the deceleration parameter $q$.

\begin{tabular}{|c|c|c|c|c|c|c|}
\hline Label & Location of $\left(x_{c}, y_{c}, z_{c}, u_{c}\right)$ & Exists for & $\Omega_{\mathrm{DE}}$ & $\omega_{\mathrm{DE}}$ & $\omega_{\text {tot }}$ & $q$ \\
\hline$A$ & $\left(\frac{\sqrt{2}}{2}, 0, \frac{\sqrt{2}}{2}, u_{c}\right)$ & $\xi<0$ & 1 & -1 & -1 & -1 \\
\hline$B$ & $\left(\frac{\sqrt{2}}{2}, 0,-\frac{\sqrt{2}}{2}, u_{c}\right)$ & $\xi<0$ & 1 & -1 & -1 & -1 \\
\hline C & $\left(-\frac{\sqrt{2}}{2}, 0, \frac{\sqrt{2}}{2}, u_{c}\right)$ & $\xi<0$ & 1 & -1 & -1 & -1 \\
\hline$D$ & $\left(-\frac{\sqrt{2}}{2}, 0,-\frac{\sqrt{2}}{2}, u_{c}\right)$ & $\xi<0$ & 1 & -1 & -1 & -1 \\
\hline $\mathscr{L}_{1}($ critical line $)$ & $\left(\sqrt{1+z_{c}^{2} \operatorname{sgn}(\xi)}, 0, z_{c}, 0\right)$ & All $\xi$ & 1 & -1 & -1 & -1 \\
\hline $\mathscr{L}_{2}($ critical line $)$ & $\left(-\sqrt{1+z_{c}^{2} \operatorname{sgn}(\xi)}, 0, z_{c}, 0\right)$ & All $\xi$ & 1 & -1 & -1 & -1 \\
\hline
\end{tabular}

TABLE 2: The eigenvalues of the linearization matrices corresponding to the critical points and lines in Table 1. Here, $\lambda_{1}=216|\xi| u_{c}^{2}+27$ and $\lambda_{2}=6144|\xi|^{3} u_{c}^{6}-1404|\xi|^{2} u_{c}^{4}+243|\xi| u_{c}^{2}$.

\begin{tabular}{lcc}
\hline Eigenvalues & $A, B, C, D$ & 0 \\
\hline$v_{1}$ & $\frac{1}{3}\left(\lambda_{1}+12 \sqrt{\lambda_{2}}\right)^{1 / 3}-\frac{\left(32|\xi| u_{c}^{2}-3\right)}{\left(\lambda_{1}+12 \sqrt{\lambda_{2}}\right)^{1 / 3}}-2$ & -3 \\
\hline$v_{2}$ & $-\frac{1}{6}\left(\lambda_{1}+12 \sqrt{\lambda_{2}}\right)^{1 / 3}+\frac{\left(32|\xi| u_{c}^{2}-3\right)}{2\left(\lambda_{1}+12 \sqrt{\lambda_{2}}\right)^{1 / 3}}-2$ \\
$v_{3}$ & $+\frac{\sqrt{3}}{2} I\left(\frac{1}{3}\left(\lambda_{1}+12 \sqrt{\lambda_{2}}\right)^{1 / 3}+\frac{\left(32|\xi| u_{c}^{2}-3\right)}{\left(\lambda_{1}+12 \sqrt{\lambda_{2}}\right)^{1 / 3}}\right)$ \\
\hline$v_{4}$ & $-\frac{1}{6}\left(\lambda_{1}+12 \sqrt{\lambda_{2}}\right)^{1 / 3}+\frac{\left(32|\xi| u_{c}^{2}-3\right)}{2\left(\lambda_{1}+12 \sqrt{\lambda_{2}}\right)^{1 / 3}}-2$ \\
& $-\frac{\sqrt{3}}{2} I\left(\frac{1}{3}\left(\lambda_{1}+12 \sqrt{\lambda_{2}}\right)^{1 / 3}+\frac{\left(32|\xi| u_{c}^{2}-3\right)}{\left(\lambda_{1}+12 \sqrt{\lambda_{2}}\right)^{1 / 3}}\right)$ \\
\hline
\end{tabular}

the linearization matrix are of opposite sign, namely, $v_{2}>0$ and $v_{3,4}<0$ or $\nu_{2}<0$ and $\nu_{3,4}>0$. On the other hand, if $v_{2}<0$ and $v_{3,4}<0$, then point $A$ is a stable point (meaning that if the universe reaches this solution, it remains there forever). Point $A$ would be corresponding to a repeller (nonvanishing eigenvalues of the corresponding linearization matrix are positive) if $v_{2}>0$ and $v_{3,4}>0$. This unstable solution is usually associated with early time dynamics. We mention that in Tables 2 and 3 the variables $\lambda_{1}, \lambda_{2}$, and $\widehat{\xi}$ are defined by

$$
\begin{gathered}
\lambda_{1}=216|\xi| u_{c}^{2}+27 \\
\lambda_{2}=6144|\xi|^{3} u_{c}^{6}-1404|\xi|^{2} u_{c}^{4}+243|\xi| u_{c}^{2} \\
\widehat{\xi}=32|\xi| u_{c}^{2}-3 .
\end{gathered}
$$

3.2. Critical Points $B, C$, and D. Like the critical point $A$, the critical points $B, C$, and $D$ exist for $\xi<0$. The cosmological properties of these dark energy dominated solutions are the same as the properties of the critical point $A$. These points can be stable, unstable, or saddle, depending on the sign of the eigenvalues of the corresponding linearization matrix. Note however that, since the variable $x$ has the sign of $H$, thus critical points with $x_{c}>0$ (points $A$ and $B$ ) correspond to expansion, while critical points with $x_{c}<0$ (points $C$ and $D$ ) correspond to contraction.

The projection of the phase space evolution on the $x-u$ plane, for the tachyonic teleparallel dark energy model with $\xi=-0.001$, has been plotted in Figure 1. This figure is plotted for the case with $y=0$. With these values in the parameter space, this figure shows that points with $x_{c}= \pm \sqrt{2} / 2 \approx 0.707$ and arbitrary $u_{c}$ can be attractors of the model.

3.3. Critical Line $\mathscr{L}_{1}$. In tachyonic teleparallel dark energy, we also have two critical lines. The critical line $\mathscr{L}_{1}$ is a dark energy dominated solution with equation of state equal to -1 . Its location is $\left(\sqrt{1+z_{c}^{2} \operatorname{sgn}(\xi)}, 0, z_{c}, 0\right)$ which is shown in Table 1. As seen from Table 2, since in this case the two 
TABLE 3: Conditions for $v_{2}, v_{3}$, and $v_{4}$ to be positive or negative. Here, $\widehat{\xi}=32|\xi| u_{c}^{2}-3$.

\begin{tabular}{lc}
\hline Eigenvalues & Condition \\
\hline$v_{2}>0$ & $\left(\lambda_{1}+12 \sqrt{\lambda_{2}}\right)^{1 / 3}>3+\sqrt{9+3 \hat{\xi}}$ or $\left(\lambda_{1}+12 \sqrt{\lambda_{2}}\right)^{1 / 3}<3-\sqrt{9+3 \hat{\xi}}$ \\
$v_{2}<0$ & $3-\sqrt{9+3 \widehat{\xi}}<\left(\lambda_{1}+12 \sqrt{\lambda_{2}}\right)^{1 / 3}<3+\sqrt{9+3 \widehat{\xi}}$ \\
$v_{3,4}>0$ & $\left(\lambda_{1}+12 \sqrt{\lambda_{2}}\right)^{1 / 3}>-6+\sqrt{36+3 \hat{\xi}}$ \\
$v_{3,4}<0$ & $\left(\lambda_{1}+12 \sqrt{\lambda_{2}}\right)^{1 / 3}<-6+\sqrt{36+3 \widehat{\xi}}$ \\
\hline
\end{tabular}

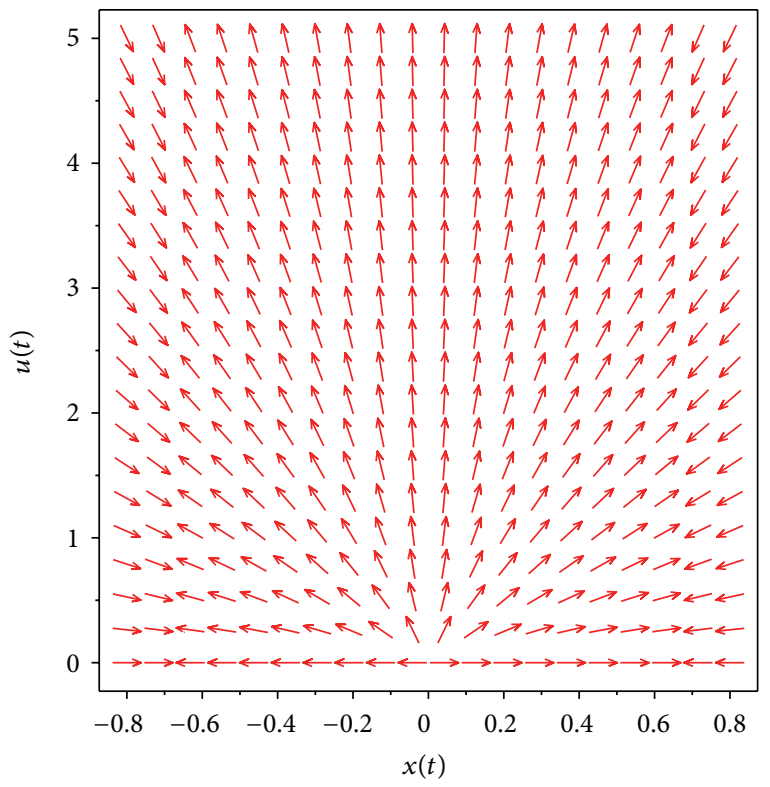

FIGURE 1: The projection of the phase space evolution on the $x-$ $u$ plane, for the tachyonic teleparallel dark energy model with $\xi=$ -0.001 . This figure is plotted for the case with $y=0$. With these values in the parameter space, this figure shows that points with $x_{c}=$ $\pm \sqrt{2} / 2 \approx 0.707$ and arbitrary $u_{c}$ can be attractors of the model.

nonvanishing eigenvalues of the perturbation matrix are negative, the critical line $\mathscr{L}_{1}$ can be a stable attractor.

3.4. Critical Line $\mathscr{L}_{2}$. This line is located at $\left(-\sqrt{1+z_{c}^{2} \operatorname{sgn}(\xi)}\right.$, $\left.0, z_{c}, 0\right)$ and the behavior of the universe in this case is like that of critical line $\mathscr{L}_{1}$. Both critical lines $\mathscr{L}_{1}$ and $\mathscr{L}_{2}$ exist for all values of nonminimal coupling parameters. The critical lines $\mathscr{L}_{1}$ and $\mathscr{L}_{2}$ have been plotted on the phase plane $x-z$ in Figure 2.

Before closing this section, let us make a comment on stability of the critical points and critical lines. Since the critical points and lines, which are obtained in our model, are nonhyperbolic (at least one of the eigenvalues has a zero real part), in general and unless some of the nonvanishing real parts of the eigenvalues are of opposite sign, no final statement can be made on their stability properties until the corresponding phase portraits are drawn.

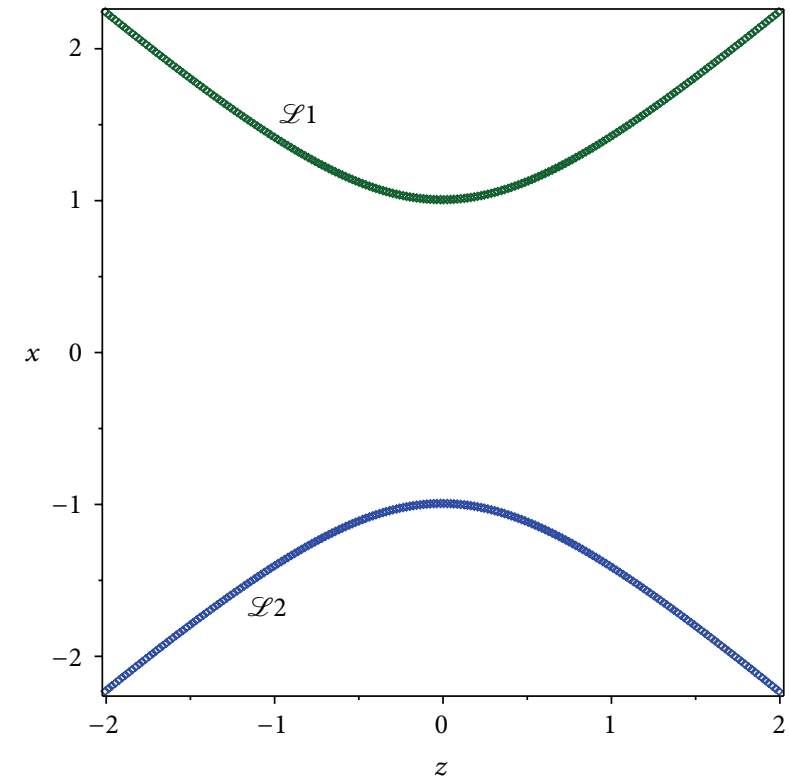

Figure 2: The critical lines $\mathscr{L}_{1}$ and $\mathscr{L}_{2}$ on the phase plane $x-z$.

\section{Conclusion}

A detailed dynamical analysis of the teleparallel dark energy has been investigated in [47]. It is shown that the universe can result in the quintessence-like, dark-energy-dominated solution similar to standard quintessence. Additionally, teleparallel dark energy possesses an additional late-time solution, in which dark energy behaves like a cosmological constant. In [48], dynamics of interacting teleparallel dark energy has been studied. The author has found that there exist only some dark-energy-dominated de Sitter attractors, and no scaling attractor has been found. In this paper, we have investigated the cosmological dynamics of tachyonic teleparallel dark energy model. The model is based on a nonminimal coupling between a noncanonical scalar field (tachyon field) in the context of teleparallel gravity. It is shown in [39] that such a model presents phantom divide crossing during its evolution in agreement with cosmological observations. We have extracted the fixed points of the model and presented the conditions for their existence and stability. Also the basic cosmological observables, namely, the total equation of state $\omega_{\text {tot }}$, deceleration parameter $q$, and dark energy equation of states $\Omega_{\mathrm{DE}}$ and $\omega_{\mathrm{DE}}$ have been calculated. We have 
found four critical points and two critical lines as shown in Table 1. We have also derived the eigenvalues corresponding to each critical points and lines in order to determine their behavior. The critical points can be attractor, unstable, or saddle depending on the sign of the eigenvalues. The critical lines could be stable attractors of the model because two nonvanishing eigenvalues of the corresponding perturbation matrix are negative. So, in all the fixed points and lines we found that stable late-time solution do exist, corresponding to an acceleration universe. Moreover, all the cases correspond to a complete dark energy dominated solution. One can also consider more complicated tachyonic potentials in order to study the phase space analysis of our model and find its fixed points.

\section{Appendix}

\section{Stability of the Critical Points}

The elements of $4 \times 4$ matrix $Q$ of the linearized perturbation equations for the real and physically meaningful critical points $\left(x_{c}, y_{c}, z_{c}, u_{c}\right)$ of the autonomous system read

$$
\begin{aligned}
& Q_{11}=-\sqrt{|\xi|} y u z^{-1}+\frac{3}{2}-\frac{9 x^{2} \sqrt{1-y^{2}}}{2+2 z^{2} \operatorname{sgn}(\xi)} \\
& +\frac{y z u \sqrt{|\xi|} \operatorname{sgn}(\xi)}{1+z^{2} \operatorname{sgn}(\xi)}\left(1+\frac{1}{\sqrt{1-y^{2}}}\right) \text {, } \\
& Q_{12}=-\sqrt{|\xi|} x u z^{-1}+\frac{3 x^{3} y}{\left(2+2 z^{2} \operatorname{sgn}(\xi)\right) \sqrt{1-y^{2}}} \\
& +\frac{x z u \sqrt{|\xi|} \operatorname{sgn}(\xi)}{1+z^{2} \operatorname{sgn}(\xi)}\left(1+\frac{1}{\sqrt{1-y^{2}}}\right) \\
& +\frac{x y^{2} z u \sqrt{|\xi|} \operatorname{sgn}(\xi)}{\left(1-y^{2}\right)^{3 / 2}\left(1+z^{2} \operatorname{sgn}(\xi)\right)}, \\
& Q_{13}=\sqrt{|\xi|} x y u z^{-2}+\frac{3 x^{3} z \operatorname{sgn}(\xi) \sqrt{1-y^{2}}}{\left(1+z^{2} \operatorname{sgn}(\xi)\right)^{2}} \\
& +\frac{x y u \sqrt{|\xi|} \operatorname{sgn}(\xi)}{1+z^{2} \operatorname{sgn}(\xi)}\left(1+\frac{1}{\sqrt{1-y^{2}}}\right) \\
& -\frac{2 x y u z^{2} \sqrt{|\xi|} \operatorname{sgn}(\xi)}{\left(1+z^{2} \operatorname{sgn}(\xi)\right)^{2}}\left(1+\frac{1}{\sqrt{1-y^{2}}}\right) \\
& Q_{14}=-\sqrt{|\xi|} x y z^{-1}+\frac{x y z \sqrt{|\xi|} \operatorname{sgn}(\xi)}{1+z^{2} \operatorname{sgn}(\xi)}\left(1+\frac{1}{\sqrt{1-y^{2}}}\right) \text {, }
\end{aligned}
$$

$Q_{21}=\frac{4 \sqrt{|\xi|}\left(1-y^{2}\right) z u}{x^{3}}$

$Q_{22}=9 y^{2}-3+4 y u \sqrt{|\xi|}\left[\frac{z}{x^{2}}-\frac{1}{z}\right]$

$Q_{23}=-2 \sqrt{|\xi|} u\left(1-y^{2}\right)\left[\frac{1}{z^{2}}+\frac{1}{x^{2}}\right]$,

$Q_{24}=2 \sqrt{|\xi|}\left(1-y^{2}\right)\left[\frac{1}{z}-\frac{z}{x^{2}}\right]$

$Q_{31}=0$,

$Q_{32}=\sqrt{|\xi|} u$,

$Q_{33}=0$,

$Q_{34}=\sqrt{|\xi|} y$,

$Q_{41}=-\frac{3 x u \sqrt{1-y^{2}}}{1+z^{2} \operatorname{sgn}(\xi)}$,

$Q_{42}=\frac{3 x^{2} y u}{\left(2+2 z^{2} \operatorname{sgn}(\xi)\right) \sqrt{1-y^{2}}}$

$+\frac{z u^{2} \sqrt{|\xi|} \operatorname{sgn}(\xi)}{1+z^{2} \operatorname{sgn}(\xi)}\left(1+\frac{1}{\sqrt{1-y^{2}}}\right)$

$+\frac{y^{2} z u^{2} \sqrt{|\xi|} \operatorname{sgn}(\xi)}{\left(1-y^{2}\right)^{3 / 2}\left(1+z^{2} \operatorname{sgn}(\xi)\right)}$

$Q_{43}=\frac{3 x^{2} z u \sqrt{1-y^{2}}}{\left(1+z^{2} \operatorname{sgn}(\xi)\right)^{2}}+\frac{y u^{2} \sqrt{|\xi|} \operatorname{sgn}(\xi)}{1+z^{2} \operatorname{sgn}(\xi)}\left(1+\frac{1}{\sqrt{1-y^{2}}}\right)$

$-\frac{2 y z^{2} u^{2} \sqrt{|\xi|} \operatorname{sgn}(\xi)}{\left(1+z^{2} \operatorname{sgn}(\xi)\right)^{2}}\left(1+\frac{1}{\sqrt{1-y^{2}}}\right)$

$Q_{44}=\frac{3}{2}-\frac{3 x^{2} \sqrt{1-y^{2}}}{2+2 z^{2} \operatorname{sgn}(\xi)}$

$$
+\frac{2 y z u \sqrt{|\xi|} \operatorname{sgn}(\xi)}{1+z^{2} \operatorname{sgn}(\xi)}\left(1+\frac{1}{\sqrt{1-y^{2}}}\right) \text {. }
$$

Examining the eigenvalues of the matrix $Q$ for each critical point, one determines its stability behavior. We mention that although the matrix $Q$ has a complicated form, inserting the explicit critical points presented in Table 1 into the elements, it takes a simple form and we can easily calculate its 
eigenvalues. The corresponding eigenvalues $v_{i}(i=1,2,3,4)$ for each critical point are presented in Table 2.

\section{Conflict of Interests}

The authors declare that there is no conflict of interests regarding the publication of this paper.

\section{References}

[1] S. Perlmutter, G. Aldering, G. Goldhaber et al., "Measurements of $\Omega$ and $\Lambda$ from 42 high-redshift supernovae," The Astrophysical Journal, vol. 517, no. 2, p. 565, 1999.

[2] A. G. Riess, A. V. Filippenko, P. Challis et al., "Observational evidence from supernovae for an accelerating universe and a cosmological constant," The Astronomical Journal, vol. 116, no. 3, p. 1009, 1998.

[3] D. N. Spergel, L. Verde, H. V. Peiris et al., "First-year Wilkinson Microwave Anisotropy Probe (WMAP) observations: determination of cosmological parameters," Astrophysical Journal, vol. 148, no. 1, pp. 175-194, 2003.

[4] E. Komatsu, J. Dunkley, M. R. Nolta et al., "Five-year wilkinson microwave anisotropy probe observations: cosmological interpretation," The Astrophysical Journal Supplement Series, vol. 180, no. 2, p. 330, 2009.

[5] M. Tegmark, M. A. Strauss, M. R. Blanton et al., "Cosmological parameters from SDSS and WMAP," Physical Review D, vol. 69, no. 10, Article ID 103501, 26 pages, 2004.

[6] U. Seljak, M. Alexey, M. Patrick et al., "Cosmological parameter analysis including SDSS Ly $\alpha$ forest and galaxy bias: constraints on the primordial spectrum of fluctuations, neutrino mass, and dark energy," Physical Review, vol. 71, no. 10, Article ID 103515, 20 pages, 2005.

[7] S. Nojiri and S. D. Odintsov, "Unified cosmic history in modified gravity: from $F(R)$ theory to Lorentz non-invariant models," Physics Reports, vol. 505, no. 2-4, pp. 59-144, 2011.

[8] T. P. Sotiriou and V. Faraoni, " $F(R)$ theories of gravity," Reviews of Modern Physics, vol. 82, no. 1, pp. 451-497, 2010.

[9] F. S. N. Lobo, "The dark side of gravity: modified theories 4 of gravity," Dark Energy-Current Advances and Ideas, pp. 173-204, 2009.

[10] S. Capozziello and V. Faraoni, Beyond Einstein Gravity, Springer, 2010.

[11] M. R. Setare and N. Mohammadipour, "Cosmography in F(G) modified gravity," General Physics. In press.

[12] B. Feng, X. Wang, and X. Zhang, "Dark energy constraints from the cosmic age and supernova," Physics Letters B, vol. 607, no. 12, pp. 35-41, 2005.

[13] Z.-K. Guo, Y.-S. Piao, X. Zhang, and Y.-Z. Zhang, "Cosmological evolution of a quintom model of dark energy," Physics Letters $B$, vol. 608, no. 3-4, pp. 177-182, 2005.

[14] B. Feng, M. Li, Y.-S. Piao, and X. Zhang, "Oscillating quintom and the recurrent universe," Physics Letters B, vol. 634, no. 2-3, pp. 101-105, 2006.

[15] W. Zhao and Y. Zhang, "Quintom models with an equation of state crossing-1," Physical Review D, vol. 73, no. 12, Article ID 123509, 12 pages, 2006.

[16] R. Lazkoz and G. León, "Quintom cosmologies admitting either tracking or phantom attractors," Physics Letters B, vol. 638, no. 4, pp. 303-309, 2006.
[17] R. Lazkoz, G. León, and I. Quiros, "Quintom cosmologies with arbitrary potentials," Physics Letters B, vol. 649, no. 2-3, pp. 103110, 2007.

[18] E. N. Saridakis and J. M. Weller, "A Quintom scenario with mixed kinetic terms," Physical Review, vol. 81, no. 12, Article ID 123523, 11 pages, 2010.

[19] E. J. Copeland, M. Sami, and S. Tsujikawa, "Dynamics of dark energy," International Journal of Modern Physics D, vol. 15, no. 11, pp. 1753-1935, 2006.

[20] L. Amendola and S. Tsujikawa, Dark Energy, Theory and Observations, Cambridge Univ. Press, Cambridge, Mass, USA, 2010.

[21] K. Bamba, S. Capozziello, S. Nojiri, and S. D. Odintsov, "Dark energy cosmology: the equivalent description via different theoretical models and cosmography tests," Astrophysics and Space Science, vol. 342, no. 1, pp. 155-228, 2012.

[22] Y.-F. Cai, E. N. Saridakis, M. R. Setare, and J.-Q. Xia, "Quintom cosmology: theoretical implications and observations," Physics Reports, vol. 493, no. 1, pp. 1-60, 2010.

[23] A. Einstein, "Translation of einstein's attempt of a unified field theory with teleparallelism," Sitzungsberichte der Königlich Preussischen Akademie der Wissenschaften zu Berlin, vol. 217, p. 224, 1928.

[24] F. W. Hehl, P. Von Der Heyde, G. D. Kerlick, and J. M. Nester, "General relativity with spin and torsion: foundations and prospects," Reviews of Modern Physics, vol. 48, no. 3, pp. 393416, 1976.

[25] K. Hayashi and T. Shirafuji, "New general relativity," Physical Review D, vol. 19, no. 12, pp. 3524-3553, 1979.

[26] J. W. Maluf, "Hamiltonian formulation of the teleparallel description of general relativity," Journal of Mathematical Physics, vol. 35, no. 1, p. 335, 1994.

[27] H. I. Arcos and J. G. Pereira, "Torsion gravity: a reappraisal," International Journal of Modern Physics D, vol. 13, no. 10, pp. 2193-2240, 2004.

[28] R. Aldrovandi and J. G. Pereira, Fundamental Theories of Physice, vol. 173, Springer, Dordrecht, The Netherlands, 2013.

[29] A. Unzicker and T. Case, "Translation of einstein's attempt of a unified field theory with teleparallelism," History and Philosophy of Physics. In press.

[30] S. Capozziello, V. F. Cardone, H. Farajollahi, and A. Ravanpak, "Cosmography in $f(T)$ gravity," Physical Review D, vol. 84, no. 4, Article ID 043527, 2011.

[31] G. R. Bengochea and R. Ferraro, "Dark torsion as the cosmic speed-up," Physical Review D, vol. 79, no. 12, Article ID 124019, 2009.

[32] E. V. Linder, "Erratum: einstein's other gravity and the acceleration of the Universe (Physical Review D-Particles, Fields, Gravitation and Cosmology (2010) 81 (127301))," Physical Review D, vol. 82, no. 10, Article ID 109902, 2010.

[33] E. V. Linder, "Erratum: einstein's other gravity and the acceleration of the Universe (Physical Review D-Particles, Fields, Gravitation and Cosmology (2010) 81 (127301))," Physical Review D, vol. 82, no. 10, Article ID 109902, 2010.

[34] J. B. Dent, S. Dutta, and E. N. Saridakis, " $f(T)$ gravity mimicking dynamical dark energy. Background and perturbation analysis," Journal of Cosmology and Astroparticle Physics, vol. 2011, article 009, 2011.

[35] K. Bamba, R. Myrzakulov, S. Nojiri, and S. D. Odintsov, "Reconstruction of $f(T)$ gravity: rip cosmology, finite-time future singularities, and thermodynamics," Physical Review D, vol. 85, no. 10, Article ID 104036, 15 pages, 2012. 
[36] K. Bamba, S. Nojiri, and S. D. Odintsov, "Effective $F(T)$ gravity from the higher-dimensional Kaluza-Klein and RandallSundrum theories," Physics Letters B, vol. 725, no. 4-5, pp. 368371, 2013.

[37] C.-Q. Geng, C.-C. Lee, E. N. Saridakis, and Y.-P. Wu, “'Teleparallel' dark energy," Physics Letters B, vol. 704, no. 5, pp. 384-387, 2011.

[38] C.-Q. Geng, C.-C. Lee, and E. N. Saridakis, "Observational constraints on teleparallel dark energy," Journal of Cosmology and Astroparticle Physics, vol. 2012, 2012.

[39] A. Banijamali and B. Fazlpour, "More on modified GaussBonnet gravity," Astrophysics and Space Science, vol. 340, no. 2, pp. 399-406, 2012.

[40] X.-M. Chen, Y. Gong, and E. N. Saridakis, "Phase-space analysis of interacting phantom cosmology," Journal of Cosmology and Astroparticle Physics, vol. 0904, 2009.

[41] I. Quiros, T. Gonzalez, D. Gonzalez, Y. Napoles, R. GarcíaSalcedo, and C. Moreno, "A study of tachyon dynamics for broad classes of potentials," Classical and Quantum Gravity, vol. 27, no. 21, Article ID 215021, 2010.

[42] J. De-Santiago, J. L. Cervantes-Cota, and D. Wands, "Cosmological phase space analysis of the F (X) - V (phi) scalar field and bouncing solutions," Physical Review, vol. D87, Article ID 023502, 2013.

[43] S. Carloni, E. Elizalde, and P. J. Silva, "An analysis of the phase space of Horava-Lifshitz cosmologies," Classical and Quantum Gravity, vol. 27, no. 4, Article ID 045004, 2010.

[44] R. J. Yang and X. T. Gao, "Phase-space analysis of a class of kessence cosmology," Classical and Quantum Gravity, vol. 28, no. 6, Article ID 065012, 2011.

[45] G. Leon and E. N. Saridakis, "Dynamical analysis of generalized Galileon cosmology," Journal of Cosmology and Astroparticle Physics, vol. 1303, 2013.

[46] K. Nozari and N. Rashidi, "Cosmological dynamics of a hybrid chameleon scenario," Advances in High Energy Physics, Article ID 803735, 10 pages, 2013.

[47] C. Xu, E. N. Saridakis, and G. Leon, "Phase-space analysis of teleparallel dark energy," Journal of Cosmology and Astroparticle Physics, vol. 2012, article 005, 23 pages, 2012.

[48] H. Wei, "Dynamics of teleparallel dark energy," Physics Letters $B$, vol. 712, no. 4-5, pp. 430-436, 2012.

[49] K. Hayashi and T. Shirafuji, "New general relativity," Physical Review D, vol. 19, no. 12, pp. 3524-3553, 1979.

[50] G. R. Bengochea and R. Ferraro, "Dark torsion as the cosmic speed-up," Physical Review D, vol. 79, no. 12, Article ID 124019, 5 pages, 2009.

[51] E. V. Linder, "Erratum: einstein's other gravity and the acceleration of the Universe [Phys. Rev. D 81, 127301 (2010)]," Physical Review D, vol. 82, no. 10, Article ID 109902, 2010.

[52] P. Wu and H. Yu, "Observational constraints on $f(T)$ theory," Physics Letters B, vol. 693, no. 4, pp. 415-420, 2010.

[53] P. Wu and H. Yu, " $f(T)$ models with phantom divide line crossing," The European Physical Journal C, vol. 71, 2011.

[54] P. Wu and H. Yu, "The dynamical behavior of $f(T)$ theory," Physics Letters B, vol. 692, no. 3, pp. 176-179, 2010.

[55] S. Chattopadhyay and U. Debnath, "Emergent universe in the chameleon, $\mathrm{F}(\mathrm{r})$ and $\mathrm{F}(\mathrm{t})$ gravity theories," International Journal of Modern Physics D, vol. 20, no. 6, p. 1135, 2011.

[56] M. Sharif and S. Rani, " $F(T)$ models within bianchi type-i universe," Modern Physics Letters A, vol. 26, no. 22, p. 1657, 2011.
[57] H. Wei, X.-P. Ma, and H.-Y. Qi, “ $F(T)$ theories and varying fine structure constant," Physics Letters B, vol. 703, no. 1, pp. 74-80, 2011.

[58] J. M. Cline, S. Jeon, and G. D. Moore, “The phantom menaced: constraints on low-energy effective ghosts," Physical Review D, vol. 70, Article ID 043543, 4 pages, 2004.

[59] E. J. Copeland, A. R. Liddle, and D. Wands, "Exponential potentials and cosmological scaling solutions," Physical Review $D$, vol. 57, no. 8, pp. 4686-4690, 1998.

[60] P. G. Ferreira and M. Joyce, "Structure formation with a selftuning scalar field," Physical Review Letters, vol. 79, no. 24, pp. 4740-4743, 1997.

[61] Y. Gong, A. Wang, and Y.-Z. Zhang, "Exact scaling solutions and fixed points for general scalar field," Physics Letters B, vol. 636, no. 5, pp. 286-292, 2006.

[62] X.-M. Chen and Y. Gong, "Fixed points in interacting dark energy models," Physics Letters B, vol. 675, no. 1, pp. 9-13, 2009.

[63] J. M. Aguirregabiria and R. Lazkoz, "A note on the structural stability of tachyonic inflation," Modern Physics Letters A, vol. 19, no. 12, pp. 927-930, 2004.

[64] E. J. Copeland, M. R. Garousi, M. Sami, and S. Tsujikawa, "What is needed of a tachyon if it is to be the dark energy?" Physical Review D, vol. 71, no. 4, pp. 1-43003, 2005.

[65] F. Huang, K. Xiao, and J.-Y. Zhu, "The dynamics of tachyon field with an inverse square potential in loop quantum cosmology," International Journal of Modern Physics D, vol. 22, no. 6, Article ID 1350030, 15 pages, 2013. 

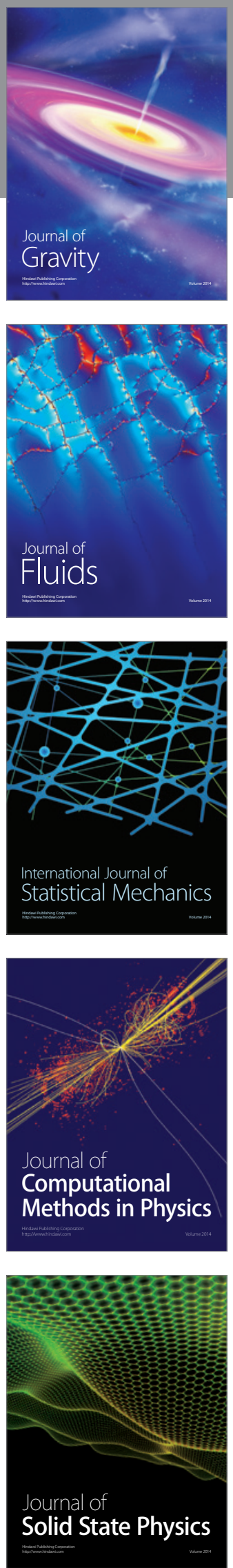

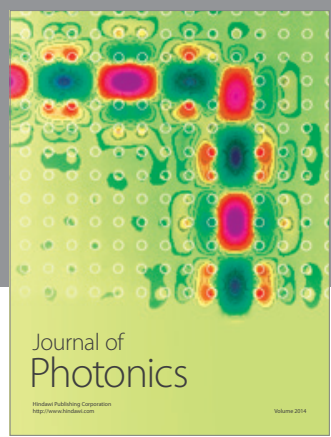

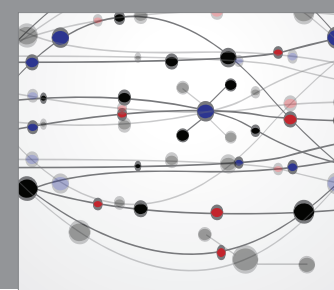

The Scientific World Journal

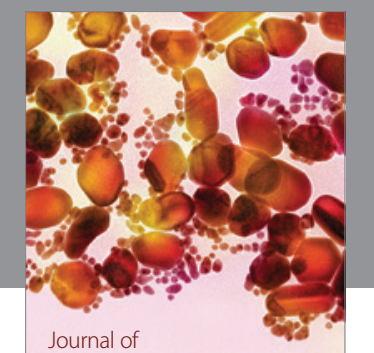

Soft Matter
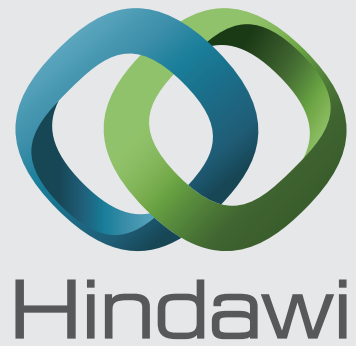

Submit your manuscripts at

http://www.hindawi.com
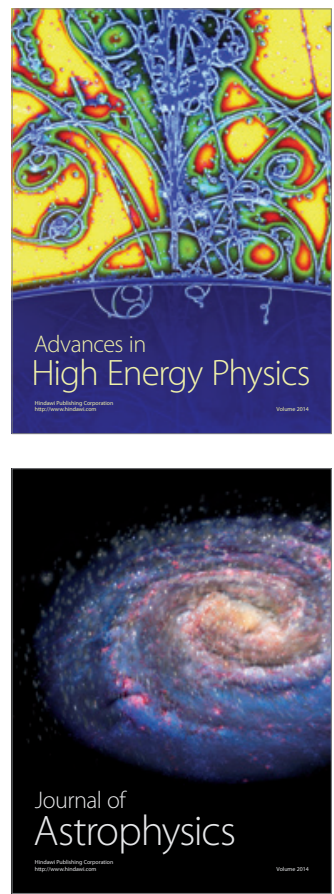
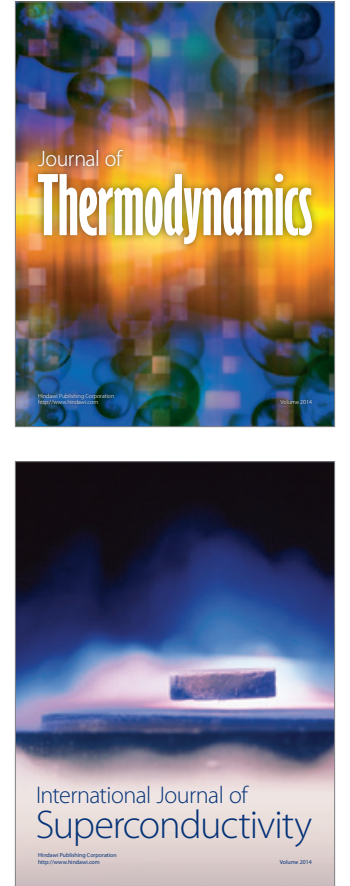
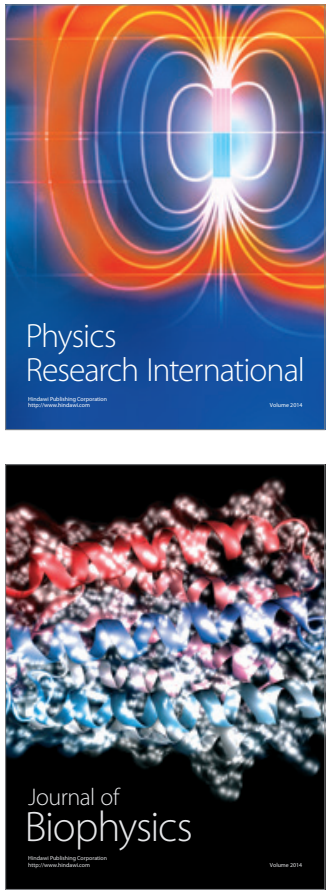
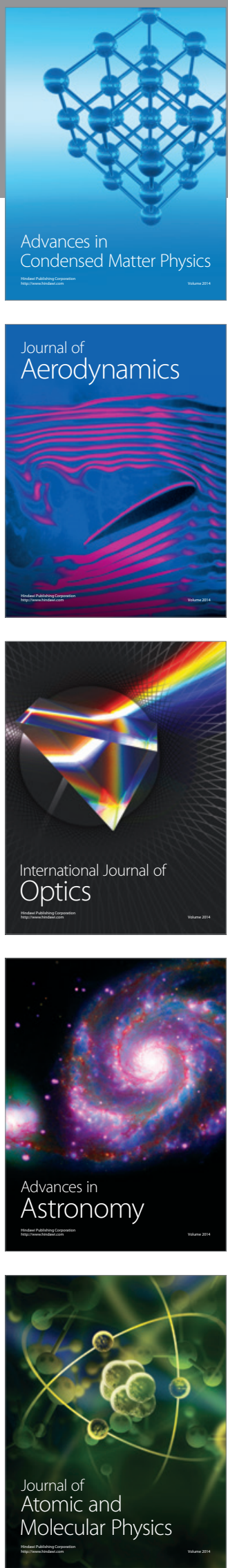